\title{
El cambiante escenario de la práctica de la Medicina y su impacto sobre la salud mental de los Médicos. Alarmantes señales para los cardiólogos.
}

Tomás Romero

Clinical Professor, University of California, School of Medicine,

San Diego, California, USA.

\section{The changing scenario of the practice of Medicine and its impact on the Physicians mental health}

The introduction of digital technology in Medicine has brought enormous diagnostic and therapeutic advances but also has impacted the practitioner's welfare and important aspects of practice such as patient-physician relations.

It has been alarming the increasing reports of physicians and nurse's burnout and associated mental disturbances such as depression and suicidal ideation. Increasing administrative burden brought to the practitioners by the need to document by digital technology patients' encounters has reduced the time of patient-physician relation and substituted by a longer time spend by the provider interacting with a computer. This represents probably one of the major causes of frustration and burnout consequences among health providers, as reported by a recent National Academy of Medicine review, a Mayo Clinic Proceedings study published in 2019, several recent Medscapes physicians surveys and by a panel discussion in the 2018 European Congress of Cardiology among many other publications. Many factors are indeed at play in this complex scenario such as government, payers, hospital facilities rules and regulations, and the way to modify them to create a more provider friendly environment may be long and difficult. Nevertheless, a first step to be considered is to reduce the administrative burden of the providers to free more time for them with their patients The future role of using diagnostic and therapeutic algorithms, some of them already available, to develop platforms of patient management with a reduced or minimal medical provider force is still uncertain and likely subject to controversial value and ethical considerations. 
La introducción de la tecnología digital y sus implicaciones tanto en el diagnóstico y tratamiento de las diversas patologías como en el acopio de la información relacionada a éstas y al manejo de los pacientes no solo ha permitido avances extraordinarios, sino que ha cambiado las modalidades de la práctica de la medicina actual.

Recientemente en Chile hubo un amplio intercambio de opiniones (“¿Puede la Medicina ser curada?”) en un órgano de difusión general ${ }^{1}$ acerca de estos cambios percibidos por médicos de diferentes especialidades y con una diversidad de puntos de vista que cubrían desde la entronización del mercado y sus efectos en la relación médico-paciente, sobrecarga de labores administrativas, hasta aspectos éticos-filosóficos del quehacer médico.

En el año 2004 se publicó en Chile un artículo basado en un estudio realizado por investigadores de los Departamentos de Psiquiatría de la Universidad de Chile y de Salud Pública de la Pontificia Universidad Católica de Chile utilizando encuestas y una técnica de análisis cualitativo a las respuestas de un grupo de 44 médicos de diferentes especialidades que, en general, coinciden con las opiniones más recientes ya citadas ${ }^{2}$.

Me referiré en esta nota específicamente a la información publicada hasta la fecha sobre el impacto de estos cambios en el alarmante costo en la salud mental de médicos en general y en particular de cardiólogos en los Estados Unidos y en la Unión Europea.

La National Academy of Medicine publicó recientemente $^{3}$ una extensa revisión sobre el tema en los profesionales de salud de Estados Unidos. Informa que más de la mitad de los médicos están afectados de síntomas de "burnout" (término adoptado universalmente en la literatura pertinente simbolizando el agotamiento del combustible mental necesario en toda actividad y que se caracteriza por pérdida de entusiasmo, satisfacción, interés y apreciación del valor del trabajo propio y de sus colegas y asociados). Los médicos trabajando en especialidades en la vanguardia de la línea de atención (servicios de emergencia, médicos generales, intensivistas, neurólogos, cardiólogos intervencionales, etc.) tienen el mayor riesgo de burnout. Después de controlar por horas de trabajo y otros factores, su prevalencia es casi el doble en los médicos que en los profesionales y trabajadores de otras actividades.

Las enfermeras presentan un grado similar de prevalencia de "burnout" y sus consecuencias que los médicos. Estudios transversales con análisis multivariado en más de 7.000 cirujanos demostraron que el burnout fue un factor predictor, independiente de errores médicos y de las probabilidades de ser objeto de una demanda judicial. Un estudio longitudinal además demostró que cada punto de aumento en el puntaje de agotamiento emocional o cada punto de disminución en la satisfacción laboral del médico estuvo asociado con un $23 \%$ y $67 \%$ de probabilidad, respectivamente, de reducir las horas de trabajo en el año siguiente, lo que significaba una pérdida de productividad al nivel nacional equivalente a eliminar la graduación de los últimos cursos de siete escuelas de medicina. Aspectos de gran alarma que afectan la salud de los médicos es la asociación independiente de burnout con alcoholismo ( $25 \%$ de aumento de las probabilidades) y $200 \%$ de mayores probabilidades de ideación suicida. Esto coincide con las estadísticas de mortalidad de la población general en los Estados Unidos que muestran que la proporción de suicidios entre los médicos de sexo masculino es $40 \%$ mayor que la del resto de los hombres, y la de los médicos de sexo femenino $120 \%$ mayor que la de la población general de mujeres.

Un estudio publicado en el Mayo Clinic Proceedings ${ }^{4}$, que incluyó a 5.197 médicos de diferentes especialidades encuestados entre los años 2017 y 2018, concluyó que $43.9 \%$ indicaban síntomas de burnout, mayor en los médicos de Emergencia, Intensivistas y Obstetras (alrededor del 50\%). En comparación con otras actividades laborales, los médicos tenían un mayor riesgo de presentarlo (OR 1.39, 95\% CI 1.26-1.54) y menor satisfacción en la integración trabajo-vida (OR 0.77, 95\% CI 0.70-0.81).

El Dr. Atul Gawande, neurocirujano del Massachusetts General Hospital y miembro del Partners Health Care que provee servicios médicos a instituciones afiliadas a Harvard y otros establecimientos académicos de New England, informa en un artículo reciente ${ }^{5}$ la frustración de la mayoría de los médicos usando el programa digital EPIC introducido en esos servicios para registro y acceso a información de los pacientes. El uso de este programa les implica emplear dos horas frente al computador por cada hora atendiendo pacientes. Gawande menciona además un estudio de la Universidad de Wisconsin en médicos de atención familiar quienes después de un aumento de su horario de atención diaria a 11 horas y media, $40 \%$ mostraron síntomas de depresión clínica y $7 \%$ ideación suicida, el doble de la prevalencia en la población laboral de los Estados Unidos.

En un estudio recientemente publicado (Mayo de 2019) 6 se estima que el costo en Estados Unidos del reemplazo por retiro y reducción de las horas de atención de los 
médicos es cercano a 4 mil millones de dólares por año. Una encuesta hecha en 2017 por Medscape ${ }^{7}$ muestra que el 52\% de los cardiólogos estaban afectados de burnout. En esta encuesta los médicos de Servicios de Emergencia y Obstetras tuvieron la mayor prevalencia (59 y 56\% respectivamente) y los Psiquiatras y Alergó$\log 0$ ( 42 y $43 \%$ ) la menor. En una escala con puntaje de 1 a 7 de las causas del burnout en los cardiólogos ( 7 = contribución significativa y $1=$ ninguna contribución), las de mayor puntaje fueron: demasiadas tareas burocráticas $=5.5$, aumento de las horas de trabajo y el uso de computadores $=5 \mathrm{y}$ entre las de menor score, demasiados casos complejos, y stress familiar $=3.6,3.2$. Un mayor porcentaje de cardiólogas $(55 \%)$ reportaron burnout en comparación a cardiólogos (51\%), pero claramente una tendencia diferente a la de una encuesta similar del año 2013 que mostró a 59\% de cardiólogas afectadas y solo un $38 \%$ de cardiólogos.

En el European Congress of Cardiology $2018^{8}$ hubo un extenso debate sobre el tema, liderado por el Dr. Anthony De María, past JACC Editor y past Chief, Sulpizio Cardiovascular Center, University of California, San Diego. En su intervención destacó que la más alta prevalencia de burnout ocurre en los cardiólogos con edades entre los 45 y 55 años, cifra particularmente alarmante ya que afecta generalmente a quienes están en su fase más productiva y cercanos a la cúspide en su especialidad. Se refirió, además, al impacto negativo de las crecientes reglas y mandatos provenientes del gobierno, de los aseguradores, y organizaciones hospitalarias y en especial a la transición a sistemas electrónicos de documentación de las atenciones lo que obliga al cardiólogo sustraer tiempo a su contacto directo con el paciente.

En resumen, son múltiples los factores que conspiran contra la salud mental de los médicos, y las vías para modificarlos y crear un entorno más amigable para los proveedores de salud pueden ser de larga y no expedita trayectoria. Sin embargo, uno de los primeros pasos factibles a tomar es simplificar la sobrecarga administrativa relacionada con el tiempo que el médico dedica a la documentación digital de sus servicios en desmedro de la relación médico-paciente.

El rol que pudiese tener en el futuro el uso de algoritmos diagnósticos y terapéuticos, ya existentes o por desarrollar, que permitieran a través de la tecnología digital crear plataformas de manejo del paciente con un empleo reducido o mínimo de recursos médicos es todavía incierto y sujeto a juicios de valor y consideraciones éticas aun no resueltas.

\section{Referencias}

1. EL MERCURIO, Letters to the Director, 2/20-3/2, 2019. www. elmercurio.com

2. HORWITZ N. The change in medical practice. Psycho-social challenges for the profession. Rev Med Chile 2004;132:76872 .

3. DYRBYE LN, SHANAFELT TD, SINSKY CA, CIPRIANO PF, BHATT J, OMMAYA A, et al. Burnout Among Health Care Professionals. A Call to Explore and Address This Under-recognized Threat to Safe, High-Quality Care. National Academy of Medicine, Perspectives I Expert Voices in Health \& Health Care. NAM.edu/Perspectives July 5, 2017.

4. SHANAFELT TD, WEST CP, SINSKY C, TROCKEL M, TUTTY M, SATELE DV, et al. Changes in Burnout and Satisfaction With Work-Life Integration in Physicians and the General US Working Population Between 2011 and 2017.Mayo Clin
Proc. https://doi.org/10.1016/j.mayocp.2018.10.023

5. GAWANDE A. The Upgrade. The New Yorker, November 12, 2018;62-73.

6. HAN S, SHANAFELT TD, SINSKY CA, AWAD KM, DYRBYE LN, FISCUS LC, et al. Estimating the Attributable Cost of Physician Burnout in the United States. Ann Intern Med. doi:10.7326/M18-1422.

7. PECKHAM C, GRISHAM S. Cardiologist Lifestyle Report 2017: Race and Ethnicity, Bias and Burnout. Medscape, January 11, 2017; 1-26. www.medscape. com/slideshow/2017-lifestyle-burnout-depression-6009235.

8. DE MARÍA A, VERHORST P, VAN GEEL D, BELL, M. Cardiologists and the Burnout Scenario. European Heart Journal 2019; 40: 5-12. doi:10.1093/eurheartj/ehy788 